\title{
Heuristics and Models for Evaluating the Usability of Security Measures
}

\author{
Denis Feth \\ Fraunhofer Institute for Experimental \\ Software Engineering IESE \\ Kaiserslautern, Germany \\ denis.feth@iese.fraunhofer.de
}

\author{
Svenja Polst \\ Fraunhofer Institute for Experimental \\ Software Engineering IESE \\ Kaiserslautern, Germany \\ svenja.polst@iese.fraunhofer.de
}

\begin{abstract}
Security mechanisms are nowadays part of almost every software. At the same time, they are typically sociotechnical and require involvement of end users to be effective. The usability of security measures is thus an essential factor. Despite this importance, this aspect often does not receive the necessary attention, for example due to short resources like time, budget, or usability experts. In the worst-case, users reject or circumvent even strong security measures and technically secure systems become insecure. To tackle the problem of unusable security measures, we developed a heuristics-based usability evaluation and optimization approach for security measures. In order to make heuristics applicable also for non-usability experts, we enrich them with information from a joint model for usability and security. In particular, this approach allows developers and administrators to perform usability evaluations and thus enables an early tailoring to the user, complementary to expert or user reviews. In this paper, we present our approach, including an initial set of heuristics, a joint model for usability and security and a set of mapping rules that combine heuristics and model. We evaluated the applicability of our approach, which we present in this paper.
\end{abstract}

\section{CCS CONCEPTS}

- Security and Privacy $\rightarrow$ Usability in security and privacy; • Human-centered computing $\rightarrow$ HCI theory, concepts and models; Heuristic evaluations.

\footnotetext{
Permission to make digital or hard copies of all or part of this work for personal or classroom use is granted without fee provided that copies are not made or distributed for profit or commercial advantage and that copies bear this notice and the full citation on the first page. Copyrights for components of this work owned by others than ACM must be honored. Abstracting with credit is permitted. To copy otherwise, or republish, to post on servers or to redistribute to lists, requires prior specific permission and/or a fee. Request permissions from permissions@acm.org.

MuC '19, September 8-11, 2019, Hamburg, Germany

(C) 2019 Association for Computing Machinery.

ACM ISBN 978-1-4503-7198-8/19/09...\$15.00

https://doi.org/10.1145/3340764.3340789
}

\section{KEYWORDS}

Usable Security, Quality Model, Usability Evaluation, Heuristic Evaluation, Human-centered Design

\section{ACM Reference Format:}

Denis Feth and Svenja Polst. 2019. Heuristics and Models for Evaluating the Usability of Security Measures. In Mensch und Computer 2019 (MuC '19), September 8-11, 2019, Hamburg, Germany. ACM, New York, NY, USA, 11 pages. https://doi.org/10.1145/3340764. 3340789

\section{INTRODUCTION}

Security and usability are important quality attributes of modern IT systems and depend strongly on each other in practice $[7,38]$. By protecting assets at different levels, security measures prevent material and non-material damage. From this point of view, security can be considered as one prerequisite for usability (e.g., in terms of trust). On the other hand, users are also part of the security chain, as security measures are often sociotechnical. Thus, considering the interdependence and trade-offs between security and usability during system development is of high importance.

\section{Motivation and Problem}

In practice, however, security measures are oftentimes difficult to understand, restrict users, and lead to additional burdens in practice $[2,3,16,17]$.

A classic negative example for the interdependency are poorly chosen password policies (e.g. regarding password length, password complexity or switching frequency) $[5,16$, 35]. While passwords must meet certain minimum requirements in order to adequately protect assets, highly restrictive password policies delay authentication and produce a high degree of mental strain on the user [16].

As a consequence, security measures lack acceptance and are frequently not used as intended. In order to overcome this issue, system developers need to consider the user's perspective on security measures early-on during the development process. Often, this is not sufficiently done in practice. 


\section{Ideas and Contributions}

We developed a tool-supported approach for evaluating and optimizing security measures with regard to their usability from the user's perspective. Our approach is based on heuristics for security and usability that are enriched with information from a joint quality model for usability and security. The approach can be applied by developers and administrators in order to improve the usability of security measures based on best practice heuristics. This allows an early tailoring to the user and raises awareness for usable security during software development. Our approach is currently in an early phase. Thus in the evaluation of our approach, we mainly focus on its general feasibility and effectiveness, rather than on the completeness and correctness of artifacts like our heuristics catalogue or mapping rules.

\section{Structure}

This paper is structured as follows: We start by introducing the overall approach in Section 2. In Section 3, we present a set of 45 heuristics for the evaluation of usability of security measures. Afterwards, we present a joint model for usability and security in Section 4. We also show how individual heuristics are mapped to different elements of the model. The combination of heuristics and model is the baseline for our tool-supported evaluation approach that is presented in Section 5. We evaluated our approach with respect to its feasibility and effectiveness. Setup and results of the evaluation are presented in Section 5. Finally, we differentiate from related work in Section 6 and conclude in Section 7.

\section{OVERALL APPROACH}

Our goal is to enable an effective and efficient evaluation and optimize security measures with respect to the usability for their users. Furthermore, we aim to empower developers and administrators to gain a better understanding of the user's objectives and needs with respect to security and privacy (e.g., security-relevant information about missing encryption [4]). The following decisions were the baseline for our approach:

(1) In general, evaluation can be divided into three categories, namely expert reviews, user reviews and collection of system feedback. We decided to focus on expert reviews. These kinds of reviews do not require a running system and typically allows the identification of a large number of problems within a short period of time.

(2) We decided to focus on the target groups "developers" and "administrators" (referred to as "evaluators" in the following) for several reasons. Although they do not necessarily have much usability expertise, they have a high knowledge about the system. Furthermore, they have a high and direct influence on how the system is built and configured, which can in turn highly influence the usability of security measures. Moreover, they are directly involved in the project anyway. Thus, it is easier and faster to task them with the evaluation, especially when compared to experienced usability and security experts.

(3) We decided for an heuristics-based evaluation approach, as hard metrics, especially for security, are quite rare and hard to apply in practice.

(4) In order to keep the user in focus, we decided for our approach to take the User-Centered Design (UCD) guidelines [26] and the Human-Centered Design (HCD) process [20] into account. HCD is an iterative process aimed at making systems usable and increasing their user experience by taking into account users' needs and requirements.

Figure 2 gives an overview on the HCD process [20] and how we support HCD's fourth phase "Evaluate Design" using heuristics. Our model respects key factors from the first three phases (e.g. activities from the first phase, privacy goals from the second phase, interaction concept from the third phase). Therefore, a full instantiation of our quality model can be achieved after the third phase. The design evaluation of the user interface and security measures is supported by enriched heuristics. The assessment of heuristics is oftentimes difficult, as evaluators need to consider a lot of background knowledge about the system under evaluation, the user groups and different context factors. Thus, we enrich our heuristics with information about the system, extracted from the system-specific model instance. Figure 1 explains this relationship.

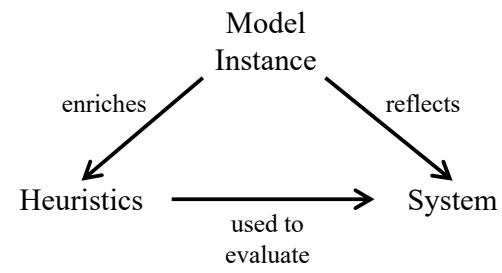

Figure 1: Relationship between System, Model Instance and Heuristics

As a concrete heuristic only relates to parts of the information about the system, the quality model is specifically filtered for each heuristic. Furthermore, our heuristics are more detailed than, for instance, the heuristics by Nielsen [24]. In this way, evaluators are supported by concrete, complementary information that support the evaluation of the heuristics. For a detailed description of the integration of our approach into HCD, please refer to [10]. 
Evaluating the Usability of Security Measures

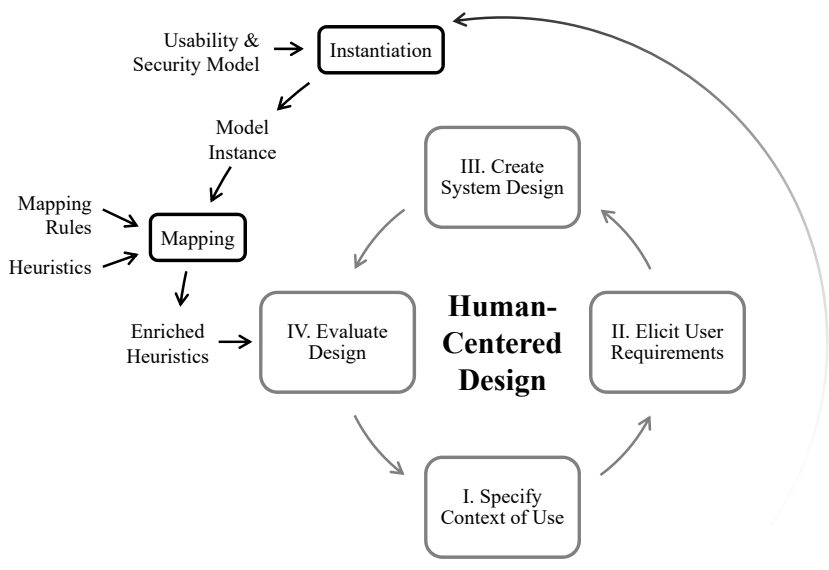

Figure 2: Overall approach and alignment to Human Centered Design

In the following sections, we present the heuristics, the model, their combination and the tool support for our approach.

\section{HEURISTICS-BASED EVALUATION}

In this chapter, the development of our heuristics is described and the final 45 heuristics are presented.

From the user's perspective, we consider four relevant goals for usable security:

(1) Data Protection: Security measures for the protection of users' personal data must be present and effective. Failures to implement effective security measures can have negative influences on user satisfaction, e.g., if data leaks occur.

(2) Efficiency: Security measures must respect the users' resources, in particular knowledge, available time and cognitive capacity. If security measures put high burdens on the users, it is likely that users reject them [30].

(3) Transparency: Security measures must be transparent for the users (i.e., comprehensible, verifiable and assessable [29]). Transparency is the basis to build an individual perception of the measures and the preservation of their privacy. It is a prerequisite for trust.

(4) Self-determination: Users must be in control of their data. This can only be achieved if users are empowered to express their privacy demands in an effective and efficient way, and if there are systems that respect or enforce these demands [31].

\section{Selection of Heuristics}

First, we collected heuristics for usable security from literature. We identified two suitable studies for our approach (cf. Section 6). Yeratziotis et. al [37] developed 13 heuristics and
MuC '19, September 8-11, 2019, Hamburg, Germany

about 90 checklist items to facilitate the evaluation of these heuristics. Realpe et al. [28] provide a set of 153 heuristics for evaluating usable security and user authentication, including some of the checklist items by Yeratziotis et al.[37]. The level of detail of the checklist items is similar to the one of the heuristics by [28]. We consider this level of detail appropriate for evaluators who do not have much experience in using heuristics, such as developers. In this paper, we consider the checklist items by Yeratziotis et al.[37] as heuristics. From the resulting 240 heuristics, we removed heuristics which were

- duplicates

- too specific to a type of system and not generalizable (e.g. "does the system support and use the HTTPS protocol”)

- too generic to have an added value and hard to specify (e.g. "does the system provide awareness and educate the user on how to complete tasks?")

- not complying to current security, usability or technical standards (e.g. "does the system avoid using more than 4-digit for the PIN")

- not answerable by developers or administrators.

Moreover, we adjusted the level of detail by refining, generalizing, or splitting heuristics so that the heuristics can be used by developers. Then, we categorized the heuristics, similar to to the categories presented by Yeratziotis et al. [37] and Realpe et al. [28]. We checked the categories for completeness and added heuristics based on our expertise in usability and security. We then prioritized the heuristics based on their value for evaluators and excluded low prioritized heuristics. Finally, we adjusted some sentence structures to stress the protagonists (the user vs. the system).

\section{Heuristics Catalogue}

Heuristics were collected and created to evaluate usability of security measures following the process described in the previous Section. In total, a catalogue of 45 heuristics was compiled in six categories.

\section{Transparency.}

(1) The user can always access security relevant information

(2) The system notifies the user about relevant changes in the security measures ${ }^{1}$

(3) The system directly informs the user about mandatory security actions he needs to perform

(4) The system provides multiple levels of security details, if the system is used both novice and expert users ${ }^{1,2}$

(5) The user can understand which security mechanisms are active

\footnotetext{
${ }^{1}$ Heuristic based on / inspired by Yeratziotis et al.[37]

${ }^{2}$ Heuristic based on / inspired by Realpe et al. [28]
} 
(6) The system only shows security information essential to the current activity ${ }^{1}$

(7) Security-relevant information is brief, easy to understand and descriptive ${ }^{1}$

(8) It is clearly stated for what purposes users' personal information is used ${ }^{1,2}$

(9) The system informs the user if it is in an insecure state ${ }^{1}$

(10) Security-related terminology is expressive and consistent within the system ${ }^{1}$

(11) Security-related terminology is consistent to standards

(12) The effect of security preferences is made clear to the user

(13) The system informs the user about the progress if there are observable delays in the system's response time to a security-related action ${ }^{1,2}$

\section{Authentication.}

(14) Authentication is always required for accessing protected or sensitive areas ${ }^{1,2}$

(15) The user can choose between different authentication options (or combinations) ${ }^{1,2}$

(16) Authentication options are designed in a way to keep the cognitive load of users low ${ }^{2}$

(17) The system enforces strict password policies that are still easy to use

(18) The user can change his password ${ }^{1,2}$

(19) The system informs the user about the password policy when he changes the password

(20) The system never shows the password by default

(21) The system informs the user whether the authentication was successful

(22) The system blocks repeated unsuccessful authentication attempts, but not after the first attempt ${ }^{1,2}$

User control and freedom.

(23) The user needs to accept the terms of use and privacy policy

(24) The user needs to confirm before data is transmitted to third parties ${ }^{2}$

(25) The user can choose between iconic and text display of security information ${ }^{1}$

(26) The system provides shortcuts for frequent security tasks ${ }^{2}$

(27) The user can customize security preferences ${ }^{1,2}$

(28) The user can reverse his security-related operations $s^{1,2}$

(29) The user can cancel security-related operations he initiated $^{1,2}$

(30) The user has to confirm actions with drastic, negative or destructive consequences ${ }^{1,2}$

(31) The user can update or delete (inaccurate) personal information ${ }^{1,2}$
(32) The system implements the principles "Security by Default" and "Privacy by default"

(33) All privacy settings are always applied to the whole system

Error recognition, diagnose, and recovering.

(34) Error messages always suggest the cause of the problem ${ }^{1}$

(35) Error messages inform the user about the severity of the problem ${ }^{1,2}$

(36) Error messages provide information about action the user should / need to take ${ }^{1}$

(37) Error messages are written in the users language ${ }^{2}$

(38) The system employs automated mechanisms to assist in the reporting of security incidents

\section{User support and documentation.}

(39) Help and documentation does not require the user to pause his task ${ }^{1}$

(40) Help and documentation follows the sequence of user security actions / use cases ${ }^{1}$

(41) Users are informed about updates in the help and documentation ${ }^{1}$

(42) Help and documentation is accessible from every screen ${ }^{1,2}$

Accessibility.

(43) The system allows to use graphical passwords to users with reading difficulties ${ }^{2}$

(44) The system allows to use textual passwords for visually impaired users

(45) The system is designed for people with the highest range of abilities

These heuristics can be refined or extended for different domains or applications, but this specialization was out of scope of our project.

\section{MODEL-BASED EVALUATION SUPPORT}

The goal of our usability and security model is to cover relevant information that helps evaluators in the assessment of the presented heuristics. In this respect, application-specific model instances are used to enrich the heuristics. As not all information is relevant for each heuristic, only relevant information is extracted from the model instance. For example, in order to evaluate heuristic 31, we need to consider information about certain use cases (like "information update"), but general security threats are most likely unimportant for that heuristic. Thus, there are rules for each heuristics, that filters less relevant information from the model instance. In the following, we describe our model and the mapping rules.

Models from the literature are usually limited to one of the two quality aspects "security" or "usability" and neglect or ignore the other aspect. Our goal was to create a common model for both aspects based on the different models 
from literature (e.g. from the Common Criteria and the ISO standards 2700x and 9241-210). Primarily, aspects of user modeling, system design (incl. user interface) and security (requirements and implementation) were considered.

\section{Usability and Security Model}

The resulting model is shown in Figure 3. To simplify the handling and explanation, we divided the model into three (overlapping) perspectives: "System Security", "System and User Context", and "System Design", which are described in this chapter. A description of the integration in the human centered design process is described in [10].

System Security. The aim of this perspective is to understand and specify the context of the system and its security measures. This context is characterized by the users, the tasks they perform, and the usage environment [11].

Information systems are defined as the sum of all components that interact to perform a given functionality [23], including applications, services or other components for information processing [19]. The information system must meet certain data protection objectives in order to protect the privacy of a user. Privacy objectives may arise from legal requirements or user preferences. To comply with legal requirements, system developers must identify the assets to be protected in accordance with legal requirements. Assets are resources that are of monetary or intangible value to at least one stakeholder [23]. For users, their assets include both personal data and the abstract good of privacy. These assets are exposed to threats-potential causes of an unwanted incident that can cause damage to an affected asset [19]. As the number and severity of threats increases, so does the risk of these assets suffering damage. In order to minimize this risk, appropriate security and privacy mechanisms (Privacy Enhancing Technology) must be integrated into the system. These mechanisms meet security objectives, including privacy objectives. A security goal describes a characteristic of a component or system that must be met to protect a user's assets from specific threats [23]. In the ISO27000 standard [19], confidentiality, integrity, availability, authenticity, accountability, non-repudiation and reliability are the most important security objectives that must be investigated in order to derive appropriate security requirements.

Once we know the goals of its users, we can begin defining use cases. Use cases describe all activities that must be performed with the help of the system to be developed. Each task in a use case must be characterized in terms of its impact on usability, accessibility, security and privacy. The results of this step can be recorded in activity descriptions, use case specifications, use case diagrams or scenario descriptions.

System and User Context. Context (cf. Figure 5) is an important factor for IT security. In different situations (i.e., contexts), users have different security demands and behave differently. Thus, the consideration of the user context is essential in this respect, since both the goals, the mental model and the available resources, as well as the behavior are influenced by the user context. Besides, also the system itself is in certain situation (or state) that depends on the context. More details about these context relations are described in [22].

System Design. The system design model describes the user's interaction with an information system.

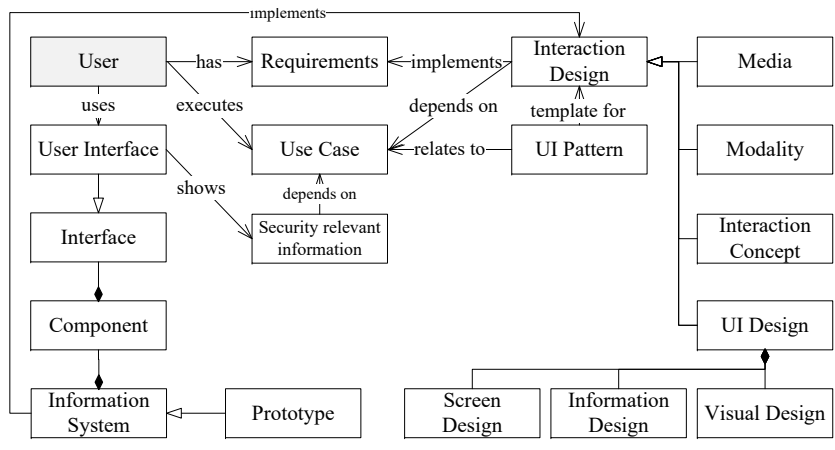

Figure 6: Model perspective "System Design"

The user and the information system are related in two ways. First, the user has requirements, which are partly implemented by the interaction design. The interaction design consists of the interaction concept, modality (i.e. channel of sensory input/output), media, and the UI design. Again, the UI design consists of several elements. These elements are information design, visual design, and screen design. The interaction design is implemented by the information system. Second, the user uses a user interface. The user interface is a kind of interface, which is a component of the information system. The user interface shows among others security related information. This information depends on the use cases, which a user executes. Also the interaction design depends on these use cases. Furthermore, UI patterns relate to use cases. UI patterns serve as a template for the interaction design.

\section{Instantiation}

Our model defines abstract classes and their relationships. In order to provide useful and concrete information for a specific application, it needs to be instantiated. The instantiation is based on the information and concepts from the first three steps of the HCD process [10] and technically produces an object model. For example, in the application "Digital Villages Platform" (cf. Section 5) the abstract class 


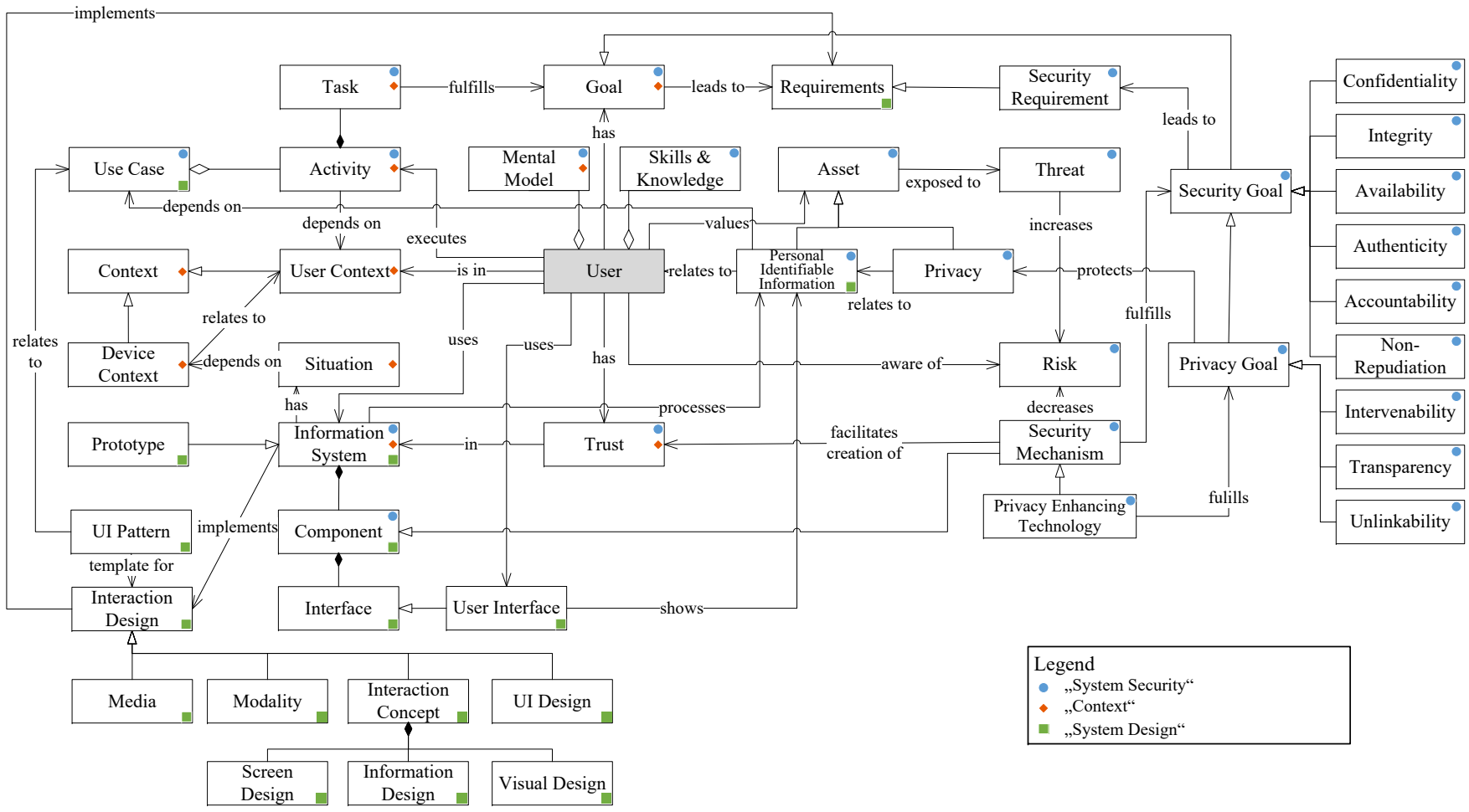

Figure 3: Joint model for usability and security aspects of the system under evaluation

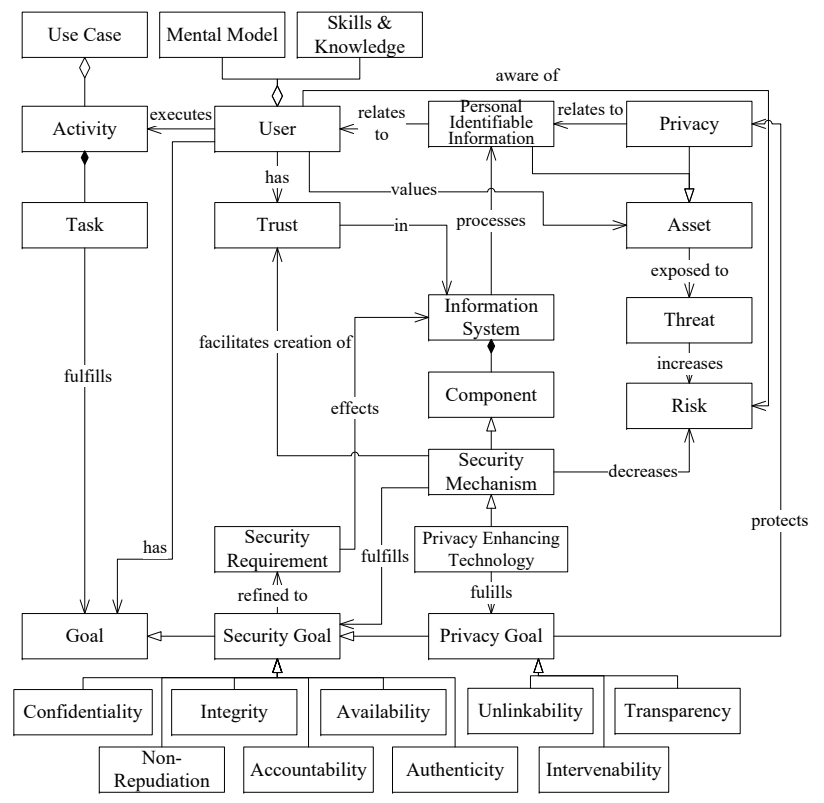

Figure 4: Model perspective "System Security"

"User" was instantiated for the concrete objects (roles) "Retailer", "Customer", "Delivery Agent", "Platform Operator", and "Support".

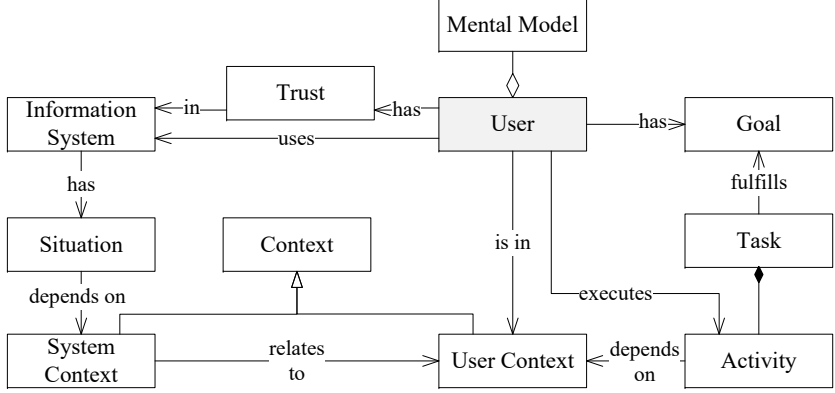

Figure 5: Model perspective "System and User Context"

\section{Mapping Heuristics and Model Elements}

In order to tailor the information from the instantiated model to the heuristics, every heuristic was mapped to a different set of model classes. Only classes that contribute valuable information for the evaluation of the heuristic were included. In the end, a filtered model instance can be generated for each heuristic. When a heuristic now relates to a "User" the heuristic can be enriched with concrete information about the instantiated users (see last section).

For reasons of space, we do not list all the mappings here. To illustrate the principle, we show two different mappings and one filtered model instance for an example application. 
The first example shows the mapping of a heuristic from the "User Control and Freedom" category (cf. Table 1). The second example shows the mapping of a heuristic from the “Transparency" category (cf. Table 2).

\begin{tabular}{|l|l|}
\hline Category & User Control and Freedom \\
\hline Heuristic & $\begin{array}{l}\text { The user can update or delete (inaccurate) per- } \\
\text { sonal information }\end{array}$ \\
\hline Mapping & $\begin{array}{l}\text { User, Information System, Component, User In- } \\
\text { terface, Intervenability, Privacy, Security Mech- } \\
\text { anism, Security Relevant Information, Use Case, } \\
\text { Activity }\end{array}$ \\
\hline
\end{tabular}

Table 1: Mapping for heuristic "Data Deletion”

\begin{tabular}{|l|l|}
\hline Category & Transparency \\
\hline Heuristic & $\begin{array}{l}\text { The system directly informs the user about } \\
\text { mandatory security actions }\end{array}$ \\
\hline Mapping & $\begin{array}{l}\text { User, Mental Model, User Interface, Trans- } \\
\text { parency, Intervenability, Skills And Knowledge, } \\
\text { UI Design, Security Mechanism, Security Rele- } \\
\text { vant Information, Use Case, Activity }\end{array}$ \\
\hline
\end{tabular}

Table 2: Mapping for heuristic "User information"

The resulting model instance for the first example for the application "IND2 UCE" (cf. Section 5) is shown in Figure 7. We additionally filtered the resulting model instance for the role "Admin". This additional filtering helps to set the focus on a particular user group and to have clearer view on it. Note that not all mapped classes are present in the diagram. This can occur if not all classes were instantiated (e.g., because some aspects are not yet defined in first HCD iterations).

\section{EVALUATION}

\section{Goals and Participants}

As our idea is in an early phase, the evaluation mainly focuses on the applicability and effectiveness of the overall approach. Aspects like the completeness of the heuristics and the correctness of the mapping are to be evaluated in the future.

The evaluation took place at the Fraunhofer IESE with seven participants (six men, median age 34), four of them IND $^{2}$ UCE developers and three "Digital Villages Platform" developers. All participants had a university degree, one participant a doctorate. The participants had medium to expert knowledge and experience in IT-security (scale 1 (no knowledge $\&$ experience) to 5 (expert knowledge \& experience), Median $=3$ ), rated their expertise in usability and quality assurance as medium (each $\mathrm{Mdn}=3$ ) and their experience and knowledge with UML class diagrams as high $(\mathrm{Mdn}=4)$. The participants evaluated the user-friendliness of the security mechanisms implemented in the application "IND ${ }^{2} \mathrm{UCE}$ " or in "LieferBar", which is part of the "Digital Villages Platform". They evaluated the application that they developed. For each application, the model was instantiated and the tool configured accordingly. Each participant worked on his own laptop and was forbidden to talk to others.

\section{Setup}

First, we informed the participants of the context and procedure. We briefly introduced the project to the participants, explained the topic and informed about the duration (approx. 2 hours) and procedure of the evaluation. The participants then signed the informed consent and filled out a demographic questionnaire.

The actual evaluation was divided into two parts. In the first part, each participant intuitively rated the user-friendliness of the security measures implemented in their application, as well as the priority given to usable security in their project. Afterwards, the participant listed and prioritized usability issues related to security they are aware of. Therefore, the participants received a form, where they could describe and prioritize the problem, but no further guidance. Finally, the participant estimated how complete his list of problems is.

In the second part of the evaluation, the participants used our tool. Participants were asked to assess the system with the heuristics ${ }^{3}$ and to use the model instance where applicable, but at least for the first three heuristics. After a participant had gone through all the heuristics, he received another questionnaire. In this questionnaire, the same questions as in the first part of the evaluation were asked. In addition, questions were asked regarding the usefulness and use of our approach in general (Technology Acceptance Model (TAM) [8]), as well as the model in particular.

Finally, we conducted a short interview with each participant about their general impression as well as advantages and disadvantages.

\section{Tool Support}

The heuristic-based evaluation can theoretically be done on paper. The manual mapping of the application-specific model instance (cf. Section 4), however, is difficult without technical support. Therefore, a prototypical tool was developed that guides the evaluator through the evaluation process and automatically filters the model instance for the current heuristic.

\footnotetext{
${ }^{3}$ We slightly changed the wording of some heuristics in retrospect (Section 3) to make them clearer. The original heuristics are available on request.
} 
In the beginning, it is necessary to configure our tool by instantiating the model for the concrete application (cf. Section 4). This needs to be done by domain and / or application experts based on the information from the HCD process.

Figure 7 shows the evaluation page for one heuristic ${ }^{4}$. On the left-hand side, heuristics have to be evaluated with "Yes", "No" or "Not applicable". On the right-hand side, the corresponding filtered model for the current heuristic is shown to support the evaluator. If a heuristic is not evaluated positively, the evaluator is asked to justify this decision. A general justification, but also a commenting of certain model elements is possible. In addition, a severity rating is assigned which should prioritize the problem (low, medium, high) from the user's point of view.

\section{Results}

After applying our approach, four participants rated the usability of the security measures in their software worse than before. This result indicates that the participants have a sharpened view of the topic after using our approach. Nevertheless, the priority, which is given to usable security in the projects, was estimated as appropriate by six participants, and as too low by one person. The estimation was exactly the same before and after applying our approach. Five participants indicated that they would use our approach in a review of their software, two were undecided. Six participants would also apply our approach for designing and planing new security measures. Among other things, the systematic approach was perceived positively.

The model was evaluated in terms of how participants used it and how useful it was for them. The participants had the task to use the model explicitly for at least the first three heuristics. For the remaining heuristics, two participants no longer used the model, two used it for up to 25 percent and three participants for 26-50 percent of the heuristics. The participants who used the model said that they found the model helpful. However, there were different opinions regarding the level of detail of the model. One participant felt that the model was too detailed for 70 percent of the heuristics, while another participant felt that the level of detail of the model was appropriate for 70 percent of the heuristics.

In addition to heuristics and model, it is generally important that the tool-support itself is usable. In the evaluation, the tool was predominantly perceived as easy to use, with the interaction with the tool being assessed as clear and understandable. The participants nevertheless stated that a certain mental effort was required. However, this effort is probably not directly related to the tool, but more to the evaluation of heuristics performed with the tool.

\footnotetext{
${ }^{4}$ The screenshot was optimized for better readability
}

In summary, we found an indication that our approach is useful in its entirety and would also be used in practice. The majority of the participants stated that they had found new problems or thought-provoking impulses which should flow into the development. However, the usefulness of the model in addition to the heuristics depended on the subjective perception of the evaluators. At this point more research is needed to achieve an ideal match between model type, model instance, heuristics and user.

\section{Threats to Validity}

Our results show first tendencies, but cannot generalized at the moment. The participant group and the set of evaluation objects were relatively small and homogeneous. Thus, we cannot make conclusions on the applicability of our approach in different project contexts and with different user groups. Additionally, learning effects cannot be fully ruled out, since participants were ask to note down usability issues in the first evaluation phase (before they applied our tool).

\section{RELATED WORK}

In general, usable security has increasingly been the focus of literature since the mid-90s. In [12], Garfinkel and Lipfort summarize the history and challenges of the topic.

Well-known case studies analyze the usability of email encryption with PGP [33, 34], file sharing with Kazaa [14] and authentication mechanisms and password guidelines $[5,9,16,21]$. However, since case studies are specific to a system, system class, or application area, they can hardly be generalized and are thus not directly applicable for a general evaluation approach like ours.

Theoretical work on the other hand $[1,6]$ includes general guidelines, checklists and design principles for usable but secure systems [13, 15, 27, 34, 36]. By their very nature, such guidelines are much abstract and therefore more difficult to implement than the results of the case studies. They focus on the development of usable security systems by supporting the developers and emphasizing the importance of taking the user into account. However, they do not adopt the user's point of view or the active involvement of users in the development process. We regard such design principles as complementary to our work.

In the field of usability, the ten heuristics developed by Nielsen [24, 25] are widely used for evaluating a systems' usability. However, these heuristics do not consider security explicitly. Therefore, they do not support evaluators in assessing usable security sufficiently.

This problem was addressed by Yeratziotis et al. [37]. The authors provide a framework for the evaluation of usable security in online social networks. This framework comprises high-level heuristics and checklist items for usable security. Users as well as experts were involved in the development of 


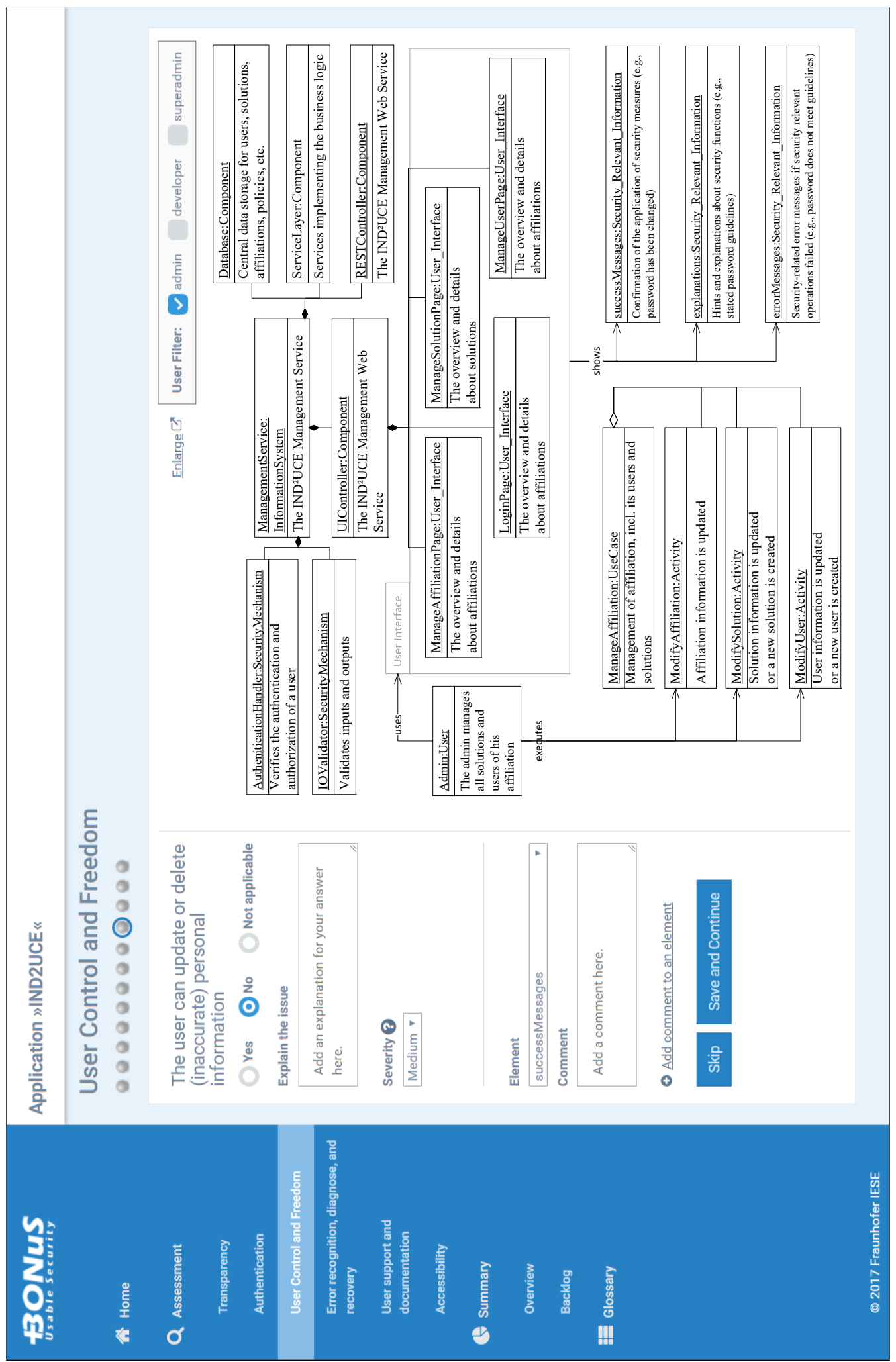

Figure 7: Heuristic with filtered model instance in the evaluation tool prototype for application "IND ${ }^{2} \mathrm{UCE}^{\text {" }}$ 
the heuristics and checklists that stem from the fields usability, security, privacy, and usable security. The usable security heuristic evaluation consists of 13 heuristics and about 90 checklist items. We made use of this catalogue for our work.

Realpe et al. [28] provide a set of 153 heuristics for usable security and user authentication, comprising the six areas usability, security and privacy, operability, accessibility, reliability, and performance. These heuristics are actually questions reflecting the same level of detail than the checklist items presented by [37]. We made use of this catalogue for our work.

Ismail et al. [18] propose a framework for assessing the transparency of cloud applications. This framework is comparable to parts of our model, but is not integrated into design and optimization processes.

\section{DISCUSSION AND CONCLUSION}

In summary, four contributions were presented in this paper. First, we created a catalogue of heuristics to evaluate the usability of security measures. The selection of the heuristics was done by the authors based on literature. They currently apply for almost all systems and are thus quite abstract. A refinement or extension of the heuristics for specific domains and technologies would help to make their application more precise. For example, usable security patterns (see e.g. [32]) should be included in the heuristics. Also, the tailoring of heuristic to concrete user groups (developers, administrations) and the extension by evaluation guidelines could help the concrete evaluators to achieve better results. This could be achieved by letting developers decide which heuristics they consider most important. However, developers from various projects would have been required to achieve a selection, which was out of our project's scope and the developers' knowledge and experience. As arbitrarily many and arbitrarily complex changes can be made in every HCD cycle, evaluators need to reevaluate every heuristic every time. In future, this could be improved by analyzing model instance changes between two HCD cycles. This information can be used to limit the reevaluation to those heuristics where changes are possible.

Second, we researched a common model for security and usability, consisting of three sub-models. All heuristics were mapped to corresponding model elements in order to tailor the model to them. As part of our future work, covering dynamic aspects would help the user to better understand the system, user, and the context. Also, the representation of the model is up to improvement. We can certainly expect developers to understand UML syntax, but other evaluator groups might not be familiar with this notation. Thus, a better (e.g., graphical or textual) presentation of the information contained by the model will probably increase the usability of our tool.
Third, we developed a web application. It allows evaluators to execute our approach. Regarding the tooling, support of the configuration (model instantiation) is our next step.

Finally, we evaluated the feasibility and effectiveness our our approach. Evaluation showed that the approach is perceived positively by developers. However, the number of participants was relatively small and only two different projects were evaluated. Thus, the results cannot be generalized at the moment. After improving the heuristics, model and tool, we plan to perform an evaluation with more participants representing various projects.

\section{ACKNOWLEDGMENTS}

The research presented in this paper is supported by the German Ministry of Education and Research (BMBF) project Software Campus (grant number 01IS12053). The sole responsibility for the content of this document lies with the authors.

\section{REFERENCES}

[1] Anne Adams and Martina Angela Sasse. 1999. Users are not the enemy. Commun. ACM 42, 12 (dec 1999), 40-46. https://doi.org/10. $1145 / 322796.322806$

[2] Ross J. Anderson. 1994. Why Cryptosystems Fail. Commun. ACM 37, 11 (Nov. 1994), 32-40. https://doi.org/10.1145/188280.188291

[3] Jim Blythe, Ross Koppel, and Sean W. Smith. 2013. Circumvention of security: Good users do bad things. IEEE Security and Privacy 11, 5 (sep 2013), 80-83. https://doi.org/10.1109/MSP.2013.110

[4] Reinhardt a. Botha, Steven M. Furnell, and Nathan L. Clarke. 2009. From desktop to mobile: Examining the security experience. Computers \& Security 28, 3-4 (may 2009), 130-137. https://doi.org/10.1016/j.cose. 2008.11.001

[5] Yee-Yin Choong and Mary Theofanos. 2015. What 4,500+ People Can Tell You - Employees' Attitudes Toward Organizational Password Policy Do Matter. In Human Aspects of Information Security, Privacy, and Trust, Theo Tryfonas and Ioannis Askoxylakis (Eds.). Springer International Publishing, 299-310. https://doi.org/10.1007/ 978-3-319-20376-8_27

[6] Lorrie Cranor and Simson Garfinkel. 2005. Security and Usability. O’Reilly Media, Inc. http://dl.acm.org/citation.cfm?id=1098730

[7] Lorrie Faith Cranor and Simson Garfinkel. 2004. Secure or usable? IEEE Security and Privacy 2, 5 (2004), 16-18. https://doi.org/10.1109/ MSP.2004.69

[8] Fred D Davis. 1993. User Acceptance of Information Technology: System Characteristics, User Perceptions and Behavioral Impacts. Int. 7. Man-Mach. Stud. 38, 3 (1993), 475-487. https://doi.org/10.1006/ imms.1993.1022

[9] Ali Mohamed Eljetlawi and Norafida Ithnin. 2008. Graphical password: Comprehensive study of the usability features of the recognition base graphical password methods. Proceedings - 3rd International Conference on Convergence and Hybrid Information Technology, ICCIT 20082 (2008), 1137-1143. https://doi.org/10.1109/ICCIT.2008.20

[10] Denis Feth, Andreas Maier, and Svenja Polst. 2017. A User-Centered Model for Usable Security and Privacy. In Human Aspects of Information Security, Privacy and Trust, Theo Tryfonas (Ed.). Springer International Publishing, Cham, 74-89.

[11] Simone Fischer-Hübner, Luigi Iacono, and Sebastian Möller. 2010. Usable Security und Privacy. Datenschutz und Datensicherheit - DuD 34 
Evaluating the Usability of Security Measures

(2010), 773-782. https://doi.org/10.1007/s11623-010-0210-4

[12] Simson Garfinkel and Heather Richter Lipford. 2014. Usable Security: History, Themes, and Challenges. Synthesis Lectures on Information Security, Privacy, and Trust 5, 2 (sep 2014), 1-124. https://doi.org/10. 2200/S00594ED1V01Y201408SPT011

[13] Simson L Garfinkel. 2005. Design Principles and Patterns for Computer Systems That Are Simultaneously Secure and Usable. Gene 31 (2005), 234-239. http://dspace.mit.edu/handle/1721.1/33204

[14] Nathaniel S Good and Aaron Krekelberg. 2003. Usability and Privacy: A Study of Kazaa P2P File-sharing. In Proceedings of the SIGCHI Conference on Human Factors in Computing Systems (CHI '03). ACM, New York, NY, USA, 137-144. https://doi.org/10.1145/642611.642636

[15] Almut Herzog and Nahid Shahmehri. 2007. Usable set-up of runtime security policies. Inf. Manag. Comput. Security 15 (2007), 394-407.

[16] Philip G. Inglesant and M. Angela Sasse. 2010. The True Cost of Unusable Password Policies: Password Use in the Wild. In Proceedings of the SIGCHI Conference on Human Factors in Computing Systems (CHI '10). ACM, New York, NY, USA, 383-392. https://doi.org/10.1145/ 1753326.1753384

[17] Philip G. Inglesant and M. Angela Sasse. 2010. The True Cost of Unusable Password Policies: Password Use in the Wild. (2010), 383392. https://doi.org/10.1145/1753326.1753384

[18] Umar Ismail, Shareeful Islam, Moussa Ouedraogo, and Edgar Weippl. 2016. A Framework for Security Transparency in Cloud Computing. Future Internet 8, 1 (2016), 5. https://doi.org/10.3390/fi8010005

[19] ISO 27001:2018. 2018. Information technology - Security techniques Information security management systems - Overview and vocabulary. Standard. International Organization for Standardization.

[20] ISO 9241-210:2010. 2010. Ergonomics of Human-System Interaction - Part 210: Human-Centred Design for Interactive Systems. Standard. International Organization for Standardization.

[21] Ian Jermyn, Alain Mayer, Fabian Monrose, Michael K Reiter, and Aviel D Rubin. 1999. The Design and Analysis of Graphical Passwords. In Proceedings of the 8th Conference on USENIX Security Symposium - Volume 8 (SSYM'99). USENIX Association, Berkeley, CA, USA, 1. http://dl.acm.org/citation.cfm?id=1251421.1251422

[22] Christian Jung and Denis Feth. 2016. Context-aware Mobile Security. International fournal on Advances in Software 9, 1 (2016), 80-94.

[23] Kompetenzzentrum für angewandte Sicherheitstechnologie (KASTEL). 2013. Begriffsdefinitionen in KASTEL.

[24] Rolf Molich and Jakob Nielsen. 1990. Improving a Human-computer Dialogue. Commun. ACM 33, 3 (1990), 338-348. https://doi.org/10. $1145 / 77481.77486$

[25] Jakob Nielsen. 1992. Finding usability problems through heuristic evaluation. In Proceedings of the SIGCHI conference on Human factors in computing systems - CHI '92. 373-380. https://doi.org/10.1145/142750. 142834
MuC '19, September 8-11, 2019, Hamburg, Germany

[26] Donald A Norman. 2002. The Design of Everyday Things. Basic Books, Inc., New York, NY, USA.

[27] Hannah Quay-de la Vallee, James M. Walsh, William Zimrin, Kathi Fisler, and Shriram Krishnamurthi. 2013. Usable security as a staticanalysis problem. In Proceedings of the 2013 ACM international symposium on New ideas, new paradigms, and reflections on programming \& software - Onward! '13. ACM Press, New York, New York, USA, 1-16. https://doi.org/10.1145/2509578.2509589

[28] Paulo C Realpe, Cesar A Collazos, Julio Hurtado, and Antoni Granollers. 2016. A Set of Heuristics for Usable Security and User Authentication. In Proceedings of the XVII International Conference on Human Computer Interaction (Interacci\&\#243;n '16). ACM, New York, NY, USA, 21:1-21:8. https://doi.org/10.1145/2998626.2998662

[29] Martin Rost and Kirsten Bock. 2011. Privacy by Design und die Neuen Schutzziele: Grundsätze, Ziele und Anforderungen. DuD - Datenschutz und Datensicherheit 35, 1 (2011), 30-35.

[30] Manuel Rudolph, Denis Feth, and Svenja Polst. 2018. Why Users Ignore Privacy Policies - A Survey and Intention Model for Explaining User Privacy Behavior. In Human-Computer Interaction. Theories, Methods, and Human Issues, Masaaki Kurosu (Ed.). Springer International Publishing, Cham, 587-598.

[31] Manuel Rudolph, Svenja Polst, and Joerg Doerr. 2019. Enabling Users to Specify Correct Privacy Requirements. In Requirements Engineering: Foundation for Software Quality, Eric Knauss and Michael Goedicke (Eds.). Springer International Publishing, Cham, 39-54.

[32] USecured. 2018. Usable Security by Design. https://www.usecured.de

[33] Alma Whitten. 1999. Why Johnny Can 't Encrypt : A Usability Evaluation of PGP 5 . 0 University of California. Science 99, August (aug 1999), 169-184. http://dl.acm.org/citation.cfm?id=1251421.1251435http: //citeseerx.ist.psu.edu/viewdoc/download?doi=10.1.1.152. $6298\{\&\}$ rep $=$ rep $1\{\&\}$ type $=$ pdf

[34] Alma Whitten. 2004. Making Security Usable. Computers Security 26, May (2004), 434-443. https://doi.org/10.1016/j.cose.2007.06.003

[35] Jeff Yan, Blackwell Alan, Ross Anderson, and Alasdair Grant. 2004. Password memorability and security: Empirical results. IEEE Security and Privacy 2, 5 (2004), 25-31. https://doi.org/10.1109/MSP.2004.81

[36] Ka-Ping Yee. 2004. Aligning security and usability. IEEE Security \& Privacy Magazine 2, 5 (sep 2004), 48-55. https://doi.org/10.1109/MSP. 2004.64

[37] Alexandros Yeratziotis, Darelle Greunen, and Dalenca Pottas. 2012. A framework for evaluating usable security: The case of online health social networks. Proceedings of the 6th International Symposium on Human Aspects of Information Security and Assurance, HAISA 2012 (2012), 97-107.

[38] Mary Ellen Zurko and Richard T. Simon. 1996. User-centered security. In Proceedings of the 1996 workshop on New security paradigms - NSPW '96. ACM Press, New York, New York, USA, 27-33. https://doi.org/10. $1145 / 304851.304859$ 